\title{
Local fields in random dielectrics: Distribution characteristics and the effects of microstructure
}

\author{
Zhe Chen* and Ping Sheng \\ Corporate Research Science Laboratories, Exxon Research and Engineering Company, Annandale, New Jersey 08801 \\ (Received 9 January 1990; revised manuscript received 11 September 1990)
}

\begin{abstract}
A generalized Onsager approach is developed for the calculation of local fields in random dielectrics. The distribution of local fields obtained is shown to display a double-peak character in general. This unexpected behavior is explained in terms of two types of local environment defined relative to the applied field. We find the characteristics of the local-field distribution to be sensitive to the microstructure of a random medium. Two types of random structures, characterized by uniform or percolative spatial correlations, were considered. The standard deviation of the distribution is shown to be directly proportional to the product of the polarizability and a parameter that measures the local anisotropy of the medium. The mean of the distribution is found to be bounded below by the Lorentz local-field value, with the fractional deviation, $S$, from the lower bound proportional to the variance $\sigma^{2}$. The same quantity $S$ is identified as the correction factor to the Clausius-Mossotti relation. We demonstrate that the extended spatial correlation has a significant effect on the values of $S$. Implications of our results, for effective-medium theories and optical properties of random small-particle systems, are discussed.
\end{abstract}

\section{INTRODUCTION}

When an electric field $\mathbf{E}$ is applied to a dielectric medium consisting of polarizable particles, the local field ${ }^{1}$ at a particular particle differs from $\mathbf{E}$ by an amount that is the sum of the dipole fields from all the other polarized particles. Due to the long-range nature of the dipole field, however, a straightforward summation of the dipolar contributions would yield conditionally convergent results. In 1909, Lorent $z^{2}$ proposed the physical concept of (what has become known as) the Lorentz cavity to evaluate the local field. This classical solution of the local-field problem, together with the associated Clausius-Mossotti (CM) relation, ${ }^{3}$ may rightly be regarded as the basis for the modern theory of dielectric materials.

In spite of its overwhelming success, however, the Lorentz theory still left some important questions about the local field unresolved. For example, whereas the Lorentz theory predicts a unique local-field value for a given medium, yet for a random medium the diversity of local environments is expected to generate a distribution of local fields. What is the shape and quantitative characteristics of this distribution? What is the relationship between the local-field distribution and the microstructure of a random medium? Although in the past decades there have been continued theoretical studies on the effects of randomness, yet the formal nature of these investigations ${ }^{4-8}$ has precluded their addressing the specific issues raised above. The physical effects of randomness on local field therefore remain today a topic to be clarified.

Another problem with the Lorentz theory is in regard to its generalization to polar media where each constituent particle not only is polarizable, but also has a permanent dipole moment $\boldsymbol{\mu}$. In that case the simple replacement of the polarizability $\alpha$ by $\alpha+\mu^{2} / k T$ in the CM relation, ${ }^{9}$ where $T$ is temperature and $k$ the Boltzmann constant, is shown to result in divergent dielectric constant at finite temperatures, a phenomenon not generally observed. $^{10,11}$ In 1936 , Onsager ${ }^{12}$ resolved this problem by proposing a division of the local field into components such that the correct contribution of the permanent dipoles may be easily isolated and evaluated. Besides creating a new theory for the local field and the dielectric constant of polar materials, Onsager's approach offers the advantage of generalizability since its steps involve only well-defined boundary-value problems.

In this work, we present a generalization of the Onsager approach for the calculation of local fields for a cluster of particles. This generalization is motivated by two considerations. First, only by explicitly taking into account the interaction between a particle and its neighbors is it possible to study the effects of randomness and their sensitivity to microstructures. Since the original Onsager calculation involves only one particle and its interaction with an effective-medium continuum, the purpose of our present study obviously requires an extension of the calculational tool. Second, it is well known that a random system is generally characterized by a correlation length $\xi$ above which the medium may be regarded as homogeneous. Therefore, in order that the calculated results may be truly representative of the system, it is necessary that we use as our calculational unit a cluster whose dimension is at least as large or larger than the correlation length. By enabling the explicit accounting of the interactions between the polarizable particles in a consistent manner, the generalized Onsager approach can thus provide a valuable tool for determining the localfield distribution and its relation to the microstructure.

Our results ${ }^{13}$ indicate that the local-field distribution is double-peaked in general. This unexpected behavior is explained in terms of two types of local environment 
defined relative to the applied field. We also find the characteristics of the local-field distribution to be sensitive to the microstructure of a random medium. First of all, the width of the distribution as characterized by the standard deviation $\sigma$ is shown to be directly proportional to the product $\alpha \eta$, where $\eta$ is a parameter that measures the local anisotropy of the medium. Second, the mean of the distribution is found to be bounded below by the Lorentz local-field value, with the fractional deviation $S$ from the lower bound proportional to the variance $\sigma^{2}$. The same quantity $S$ is also identified as the correction factor to the Clausius-Mossotti relation. For fractal or growth aggregates, the value of $S$ is especially large, implying that for these systems the Lorentz local field and its associated dielectric constant prediction are poor approximations to their true values.

In what ensues, Sec. II describes the Onsager approach and its generalization to random media. The local-field distribution and its relationship to the geometric characteristics of a random medium are presented in Sec. III. This is followed by a discussion on some specific questions in Sec. IV. Section V presents some implications of our results for effective-medium theories. We conclude by addressing further topics to be studied.

\section{FORMULATION}

\section{A. Onsager's reaction-field approach}

In an effort to resolve the difficulty of polarization catastrophy encountered in the Lorentz theory, Onsager ${ }^{12}$ proposed in 1936 a new approach for studying the local field in polar liquids. The geometry of his approach consists of a molecule placed inside a spherical cavity of radius $a$, which is in turn embedded in an effective dielectric continuum. This is depicted in Fig. 1.

When an external electric field is applied to the system, Onsager divided the local field at the molecular position

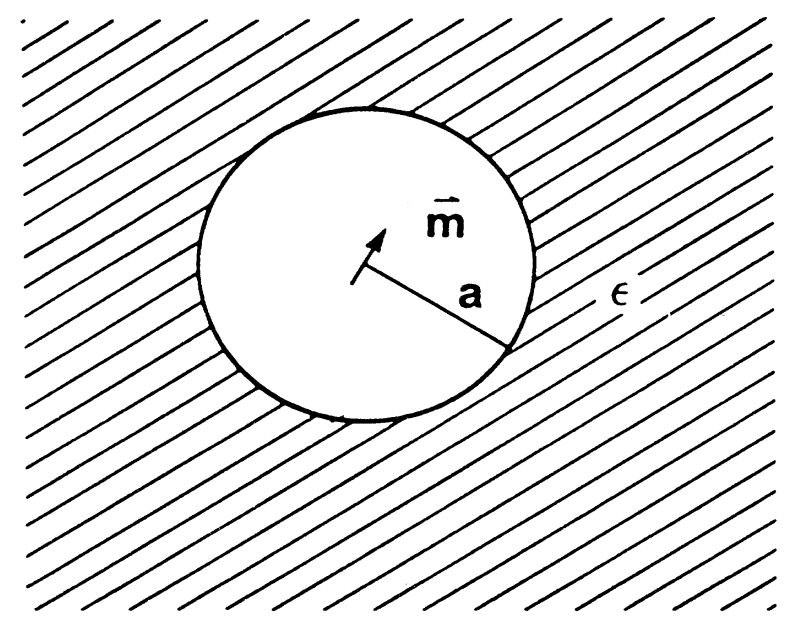

FIG. 1. Schematic sketch of the geometry used in the Onsager calculation. The cavity size is set by the averaged molecular volume so as to recover the Lorentz local field value for nonpolar molecules. (center of the cavity) as a sum of two contributions. One is due to the polarization charge on the cavity wall in the absence of the molecule, and the other is the reaction field arising from the additional polarization of the surrounding dielectric continuum due to the polarized molecule. Both fields can be obtained by solving simple electrostatic boundary-value problems.

To calculate the first part of the local field we need only to solve the Laplace equation for the electric potential inside the empty cavity with an applied external field. The relevant equations and boundary conditions are

$$
\begin{aligned}
& \nabla^{2} \phi_{\text {in }}=\nabla^{2} \phi_{\text {out }}=0, \\
& \phi_{\text {in }}=\phi_{\text {out }} \text { and } \frac{\partial \phi_{\text {in }}}{\partial r}=\epsilon \frac{\partial \phi_{\text {out }}}{\partial r} \text { at } r=a, \\
& \phi_{\text {in }} \text { is finite at } r=0,
\end{aligned}
$$

and

$$
\phi_{\text {out }} \rightarrow-E r \cos \theta \text { as } r \rightarrow \infty \text {. }
$$

In Eqs. (1), we have set the $z$ axis in the applied field direction. It is straightforward to obtain the cavity field $\mathbf{G}$ as

$$
\mathbf{G}=-\left.\nabla \phi_{\text {in }}\right|_{r=0}=\frac{3 \epsilon}{2 \epsilon+1} \mathbf{E} .
$$

The reaction field, as coined by Onsager, can be calculated by replacing the polarized molecule back into the cavity and solving for the potential without the external field. Mathematically, it differs from Eqs. (1) only in the boundary conditions (1c) and (1d), which are replaced by

$$
\phi_{\text {in }}=\frac{m \cos \theta}{r^{2}} \text { as } r \rightarrow 0
$$

and

$$
\phi_{\text {out }} \rightarrow 0 \text { as } r \rightarrow \infty
$$

where $\mathbf{m}\left(=\boldsymbol{\mu}+\mathbf{p}_{\text {induced }}\right)$ denotes the total dipole moment of the molecule. Solving the Laplace equation with the above boundary conditions yields

$$
\mathbf{R}=-\left.\nabla\left(\phi_{\text {in }}-\phi_{\text {dipole }}\right)\right|_{r=0}=\frac{2}{a^{3}} \frac{\epsilon-1}{2 \epsilon+1} \mathbf{m},
$$

where $\phi_{\text {dipole }}$ is the potential produced by a dipole $\mathbf{m}$ in vacuum. Hence the local field is

$$
\mathbf{E}_{L}=\mathbf{G}+\mathbf{R}=\frac{3 \epsilon}{2 \epsilon+1} \mathbf{E}+\frac{2}{a^{3}} \frac{\epsilon-1}{2 \epsilon+1} \mathbf{m} .
$$

The division of the local field into the cavity field and the reaction field is an important observation because $\mathbf{R}$ [Eq. (4)] is always parallel to the direction of the dipole moment. For a polar molecule with $\alpha=0$, this field therefore will not affect the spatial orientation of the molecule since it does not exert a torque on the dipole. This has significant consequences for the predicted dielectric behavior of polar liquids, as was clearly shown in Onsager's paper. ${ }^{12}$ In particular, the problem of polarization catastrophy is absent in Onsager's formulation.

The gist of Onsager's approach is the realization that a 
dipole always experiences a reaction field arising from the polarization of its neighbors by the dipole itself. Replacement of the surrounding neighbors by an effectivemedium turns the calculation of the reaction field into a well-defined boundary-value problem. It is this aspect that makes the approach mathematically generalizable.

\section{B. Generalized Onsager's approach}

A random medium is characterized by a spatial correlation length $\xi$ which is typical of the size of the inhomogeneities in the medium. The medium may be regarded as homogenenous on scales larger than the correlation length, but is inhomogeneous on scales less than $\xi$. The physical properties of a medium are known to be influenced by the nature of the correlation. ${ }^{14}$ To take into account explicitly this microstructure in our calculation of local fields, we must therefore use a cluster of particles whose dimension is as large or larger than the correlation length. We thus introduce a spherical cavity with radius $a \geq \xi$ and replace the medium outside the cavity by an effective dielectric continuum with a dielectric constant $\epsilon$ to be determined self-consistently. Since the interactions between the particles inside the cavity are treated exactly, the effects of microstructure are thus included.

For the $N$ particles inside the cavity, the local field at each particle is a sum of four contributions. First, there is the cavity field $\mathbf{G}$, which is the same for every particle. Then there is the reaction field $\mathbf{R}\left(\mathbf{r}_{i}, \mathbf{r}_{i}\right)$ due to the polarization of the continuum by the particle itself. In contrast to the Onsager theory, the position of the particle is not aways at the cavity center. In addition to these two contributions, there are the dipolar fields $\mathbf{D}\left(\mathbf{r}_{i}, \mathbf{r}_{j}\right)$ produced by all the other particles in the cavity. This contribution is absent in Onsager's theory since he considered only one particle. The last contribution is the reaction fields $\mathbf{R}\left(\mathbf{r}_{i}, \mathbf{r}_{j}\right)$ due to all the other particles. Therefore the local field $\mathbf{E}_{L}$ may be expressed as

$$
\mathbf{E}_{L}\left(\mathbf{r}_{i}\right)=\mathbf{G}+\sum_{\substack{j=1 \\ j \neq i}}^{N} \mathbf{D}\left(\mathbf{r}_{i}, \mathbf{r}_{j}\right)+\sum_{j=1}^{N} \mathbf{R}\left(\mathbf{r}_{i}, \mathbf{r}_{j}\right) .
$$

Equation (6) represents a group of $3 N$ equations for the local fields which must be solved simultaneously.

To complete the formulation, we need a relation to determine the dielectric constant of the effective-medium self-consistently. Since the system is homogeneous over the scale larger than the correlation length, we may consider the medium as composed of clusters of size $a \geq \xi$. The medium dielectric constant $\epsilon$ can then be determined from the coherent-potential approximation ${ }^{15}$ of the vanishing average forward-scattering amplitude. In the static limit (incident wavelength $\lambda \rightarrow \infty$ ), this condition. reduces to the requirement that the averaged dipole moments of the cavity equal zero, i.e.,

$$
\langle\text { cavity dipole moment }\rangle=0 \text {, }
$$

where the symbol \langle\rangle denotes configurational average. Equations (6) and (7) constitute the generalized Onsager approach.

For nonpolar systems whose constituents can be approximated by isotropic point dipoles, the dipolar field
$\mathbf{D}\left(\mathbf{r}_{i}, \mathbf{r}_{j}\right)$ may be written as

$$
\mathbf{D}\left(\mathbf{r}_{i}, \mathbf{r}_{j}\right)=\frac{3 \mathbf{r}_{i j} \mathbf{r}_{i j}-r_{i j}^{2} \overleftrightarrow{\mathbf{I}}}{r_{i j}^{5}} \cdot \mathbf{m} \equiv \overleftrightarrow{\mathbf{T}}_{i j} \cdot \mathbf{m},
$$

where $\mathbf{r}_{i j}=\mathbf{r}_{i}-\mathbf{r}_{j}$ and $r_{i j}=\left|\mathbf{r}_{i j}\right|$. The reaction field $\mathbf{R}\left(\mathbf{r}_{i}, \mathbf{r}_{j}\right)$, with arbitrary $\mathbf{r}_{i}$ and $\mathbf{r}_{j}$, and the electric potential outside the cavity can be obtained by solving an electrostatic boundary-value problem as shown in the Appendix. By substituting Eqs. (A17) and (A21) into Eqs. (6) and (7) and using $\mathbf{p}=\alpha \mathbf{E}_{L}$, we obtain the desired equations for the local fields and the dielectric constant,

$$
\begin{aligned}
& \mathbf{E}_{L}\left(\mathbf{r}_{i}\right)=\frac{3 \epsilon}{2 \epsilon+1} \mathbf{E}\left(\mathbf{r}_{i}\right)+\sum_{j=1}^{N} \overleftrightarrow{\mathbf{S}}\left(\mathbf{r}_{i}, \mathbf{r}_{j}, \epsilon\right) \cdot \mathbf{E}_{L}\left(\mathbf{r}_{j}\right), \\
& \frac{\epsilon-1}{4 \pi}=\rho \alpha\left\langle\frac{1}{N} \sum_{i=1}^{N} \mathbf{E}_{L}\left(\mathbf{r}_{i}\right) \cdot \mathbf{E} / E^{2}\right\rangle,
\end{aligned}
$$

where $\mathbf{E}$ is the macroscopic field and $\rho$ is the number density of the dipoles. The scattering matrix $\overleftrightarrow{\mathbf{S}}$ in Eq. (9) includes both the reaction field and the dipolar field:

$S_{\mu \nu}\left(\mathbf{r}_{i}, \mathbf{r}_{j}, \epsilon\right)=r_{\mu \nu}\left(\mathbf{r}_{i}, \mathbf{r}_{j}, \epsilon\right)+d_{\mu v}\left(\mathbf{r}_{i}, \mathbf{r}_{j}\right)\left(1-\delta_{i j}\right)$,

with

$$
\begin{gathered}
r_{\mu \nu}\left(\mathbf{r}_{i}, \mathbf{r}_{j}, \epsilon\right)=\alpha \sum_{l=0}^{\infty} \sum_{m=-l}^{l} c_{l m}(\epsilon)\left[\mathbf{q}\left(l, m, \mathbf{r}_{i}\right)\right]_{\mu} \\
\times\left[\mathbf{q}\left(l, m, \mathbf{r}_{j}\right)\right]_{v}^{*}, \\
d_{\mu v}\left(\mathbf{r}_{i}, \mathbf{r}_{j}\right)=\alpha \frac{3\left(\mathbf{r}_{i}-\mathbf{r}_{j}\right)_{\mu}\left(\mathbf{r}_{i}-\mathbf{r}_{j}\right)_{v}-\left|\mathbf{r}_{i}-\mathbf{r}_{j}\right|^{2} \delta_{\mu v}}{\left|\mathbf{r}_{i}-\mathbf{r}_{j}\right|^{5}},
\end{gathered}
$$

where

$$
\begin{aligned}
& \mathbf{q}\left(l, m, \mathbf{r}_{i}\right)=\nabla\left[\left(\frac{\left|\mathbf{r}_{i}\right|}{a}\right)^{l} Y_{l m}\left(\theta_{i}, \phi_{i}\right)\right], \\
& c_{l m}(\epsilon)=\frac{4 \pi}{2 l+1} \frac{(\epsilon-1)(l+1)}{l+(l+1) \epsilon} \frac{1}{a}
\end{aligned}
$$

Here $\mu, v=1,2,3$, denote the three Cartesian components of the field, and $\delta_{i j}$ is the Kronecker delta function. It should be noted that for a single particle in a cavity Eq. (9) correctly reduces to the Onsager result.

A closer examination of Eq. (10) reveals that it is identical to the constitutive relation in the electromagnetic theory, ${ }^{16} P=(\epsilon-1) / 4 \pi E$, since $P$, by definition, is the dipole moment per unit volume, i.e., $P \equiv \rho\langle p\rangle$ $=\rho\left\langle(1 / N) \sum_{i}^{N} \alpha E_{L}\right\rangle$.

Equations (9) and (10) are the basic equations of the generalized Onsager approach for nonpolar media. Together, they represent $3 N+1$ simultaneous equations. To obtain the local field and the dielectric constant $\epsilon$, we first assume an initial trial dielectric constant from the $\mathrm{CM}$ relation to solve the $3 N$ simultaneous equations [Eq. (9)] for the local fields. With the calculated local-field values, a new dielectric constant $\epsilon$ is obtained from Eq. (10). This new $\epsilon$ is substituted back into Eq. (9), and the above process is repeated until consistency is obtained. This usually takes 3-8 iterations. As the polarizability 
decreases, fewer iteration steps are needed for convergence.

With the local fields calculated for each configuration as described above, the calculation is repeated for many configurations and the configuration-averaged value of the local field is then used to get a dielectric constant from Eq. (10). Using the dielectric constant as input, we solve for local fields once again. Such iteration is continued until the averaged local field is consistent with the dielectric constant. Usually 2-3 such iteration steps are sufficient.

Since the cavity is only a contraption of our model, for particles lying close to the cavity wall their local fields would be distorted by the discontinuous change in the geometric environment. To reduce this boundary effect, we introduce a transitional region around the cavity wall: if $a-\delta \leq\left|\mathbf{r}_{i}\right| \leq a+\delta$, where $\delta$ is one nearest-neighbor distance, then particle $i$ is assigned the average local-field value at each iteration stage. It should be noted that we have created a region even outside the cavity wall. The boundary-layer particles interact with the particles inside the sphere of $r \leq a-\delta$ depending on whether they are inside or outside the cavity boundary. For a particle outside the cavity but inside the boundary region, it interacts with the inside particles via only the dipolar field. For a particle inside the cavity boundary, however, it interacts with not only the dipolar field but also the reaction field. The local-field values of the particles in the transitional region are excluded in our statistical analysis of the local-field distributions. With this prescription, our calculation correctly reproduces Lorentz's result for a fully occupied $(\rho=1)$ cubic lattice that consists of up to 27 particles. The local-field distribution in this case is sharply peaked at the Lorentz local-field value as would be expected. This check illustrates the correctness of our generalized Onsager approach in the well-known ordered case. In the next section, we will show that it also yields correct local-field values in the disordered case.

\section{NUMERICAL RESULTS AND DISCUSSIONS}

Two types of random media are considered. One is denoted as the uniform random media where every position $\mathbf{r}$ in space is occupied with the same probability, and the pair correlation length $\xi$ is proportional to $\rho^{-1 / 3}$. The other is the much studied percolative random structures, ${ }^{17}$ where the particles are required to be connected. Here the correlation length $\xi$ is known to vary as $\xi \sim\left(p-p_{c}\right)^{-v}$ for $p$ close to the percolation threshold $p_{c}$. The exponent $v$ is close to $\frac{4}{3}$ for $3 d$ site percolation. For $p \sim 1, \xi$ is on the order of the averaged nearest-neighbor distance and the system is similar to the uniform random media. For $p \rightarrow p_{c}$, $\xi$ diverges and the system exhibits fractal symmetry. ${ }^{18}$ The local environments in this limit are expected to be more anisotropic than that of a uniform random medium. It is therefore interesting to compare the local-field distribution characteristics between these two media. Since the cluster size is limited by the computer capacity, our calculations can only be performed for $p$ not too close to $p_{c}$. Nonetheless, results of such calculations already demonstrate behaviors which

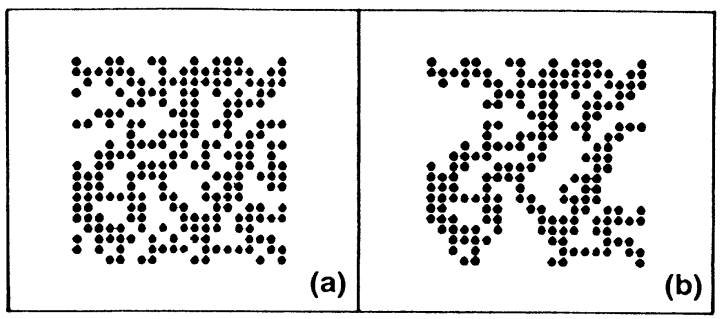

FIG. 2. (a) Two-dimensional sample of a uniform random system with particle density $\rho=0.6$. (b) Connected part of (a). It is clear from the plot that the local environment of (b) is more anisotropic than that of the uniform system (a).

are distinct from that of uniform random media.

The position of our random point particles are generated on a simple cubic lattice. Effects from the underlying lattice will be discussed in Sec. IV. To generate a uniform random medium, we start with a fully occupied lattice and randomly take out particles until a desired number density of the remaining is reached [a $2 d$ sample with $\rho=0.60$ is shown in Fig. 2(a)]. The percolative random structure is obtained by further taking out the isolated clusters from an otherwise uniform random medium, preserving only the connected part [Fig. 2(b) is obtained by retaining the connected structure of Fig. 2(a)]. To guarantee a connected structure, the occupation probability $p$ used to obtain the uniform random medium must be greater than the percolation threshold $\left(p_{c}=0.331\right.$ for $3 d$ site percolation; and $p_{c}=0.591$ for the $2 d$ case). It should be noted that $p=\rho$ for the uniform random medium but $\rho \neq p$ for the percolative structures. In fact, $\rho \rightarrow 0$ as $p \rightarrow p_{c}$. By examining Figs. 2(a) and 2(b), it is apparant that the local environment in a percolative structure is more anisotropic than that of the uniform random media. In each of our calculations the radius $a$ of the spherical cavity is always chosen so as to make the density inside the cavity as close as possible to that of the mean.

\section{A. Local-field distribution}

Figure 3 displays some of our calculated local-field distributions. Figures $3(\mathrm{a})-3(\mathrm{c})$ are for uniform random media with three different number densities. Figure 3(d) is a typical plot for a percolative random structure [with a number density of 0.261 , to be compared with Fig. 3 (c)]. It is clear that the distributions exhibit a doublepeak ${ }^{19}$ character in general. The single peak of Fig. 3(b) is interpretable as the merge of two peaks. The relative peak strength is noted to depend strongly on the number density in the uniform random system. For the local-field components perpendicular to the applied field, the distribution is found to be generally Gaussian centered at zero with a small distribution width, on the order of $0.01 E$. At a fixed number density, the separation between the two peaks is noted to vary linearly as a function of particle polarizability. Such dependence is illustrated in Fig. 4 for the $\rho=0.1$ uniform random medium with three different polarizabilities. 


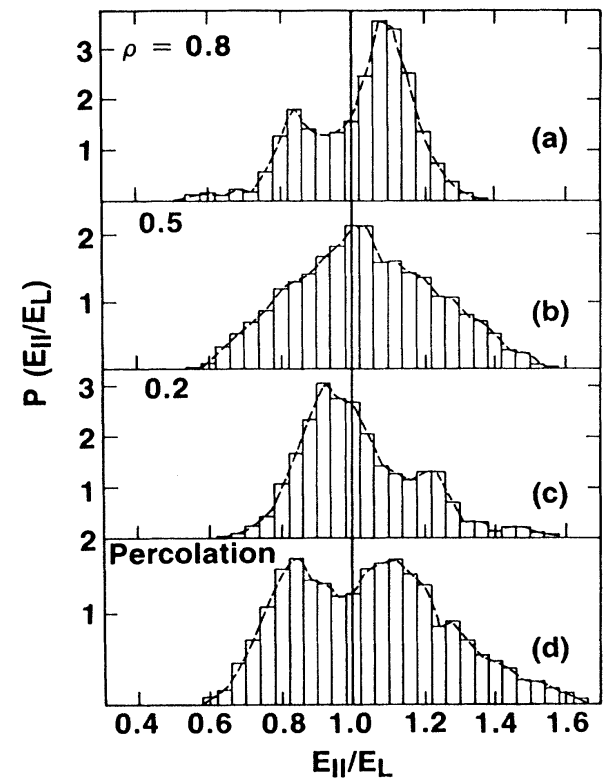

FIG. 3. Local-field distribution $P$ as a function of $E_{\|} / E_{L}$, where $E_{\|}$is defined as the component of the local field parallel to the macroscopic applied field. (a)-(c) are for uniform random media with different densities. (d) is for a random percolative structure. The number of particles used to obtain each distribution is (a) 2593, (b) 3117 , (c) 3103 , and (d) 5613.

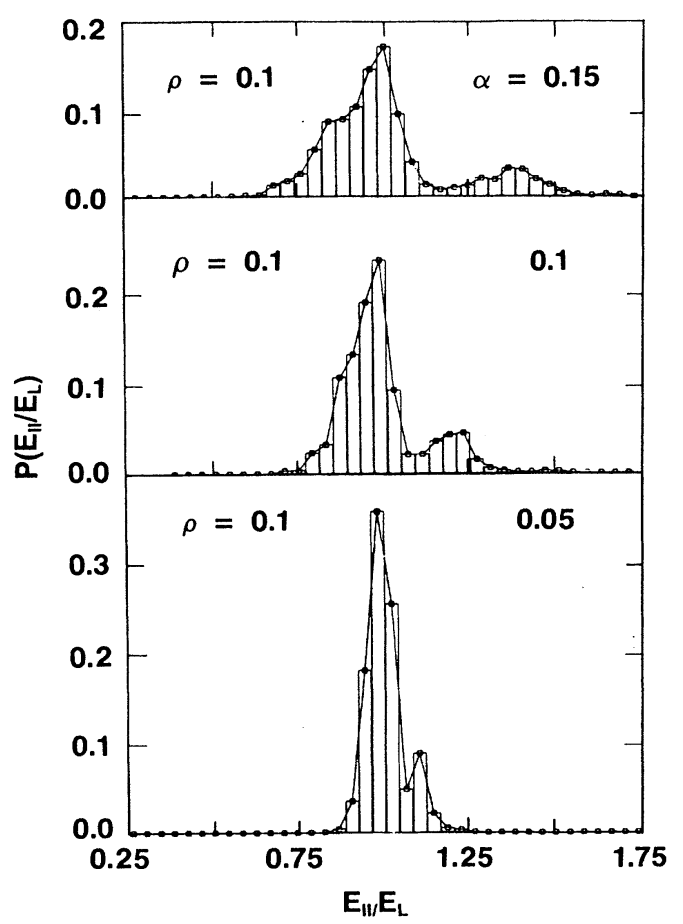

FIG. 4. Illustration of the local-field distribution $P$ for three different polarizabilities. The medium is uniformly random with a number density of 0.2 .
Before entering into the discussion on the distribution characters, it is important to ascertain that our calculational method obtains the correct local-field statistics for particles near the cavity boundary. Since the effect of the artificial cavity boundary is expected to be small for particles far away from the boundary, in Fig. 5 we directly compare the distribution results obtained from only those particles near the cavity center to that obtained from those particles close to the boundary. Figure 5(a) shows a distribution of local fields for all the particles inside the cavity ( $r<a-\delta$ with $a \sim 6.5$ and $\delta=0.5$ in units of lattice constant). The local-field distribution of particles close to the cavity center $(r<a-3.0)$ is plotted in Fig. 5(b), and Fig. 5(c) is that for particles near the boundary $(a-1.5 \leq r<a-\delta)$. Inspection of Figs. 5(a) $-5(\mathrm{c})$ assures us that the local-field distribution for particles near the cavity center is the same as that for particles close to the cavity wall.

The unexpected double-peak character of the localfield distribution can be qualitatively understood by considering the nearest-neighbor interactions. For a typical particle (denoted as $O$ in Fig. 6) in the system, it has six possible nearest-neighbor sites. When an external electric field (along the $\widehat{\mathbf{z}}$ direction) is applied to the system, it not only induces a dipole moment at each occupied site but also separates the six possible nearest-neighbor sites into

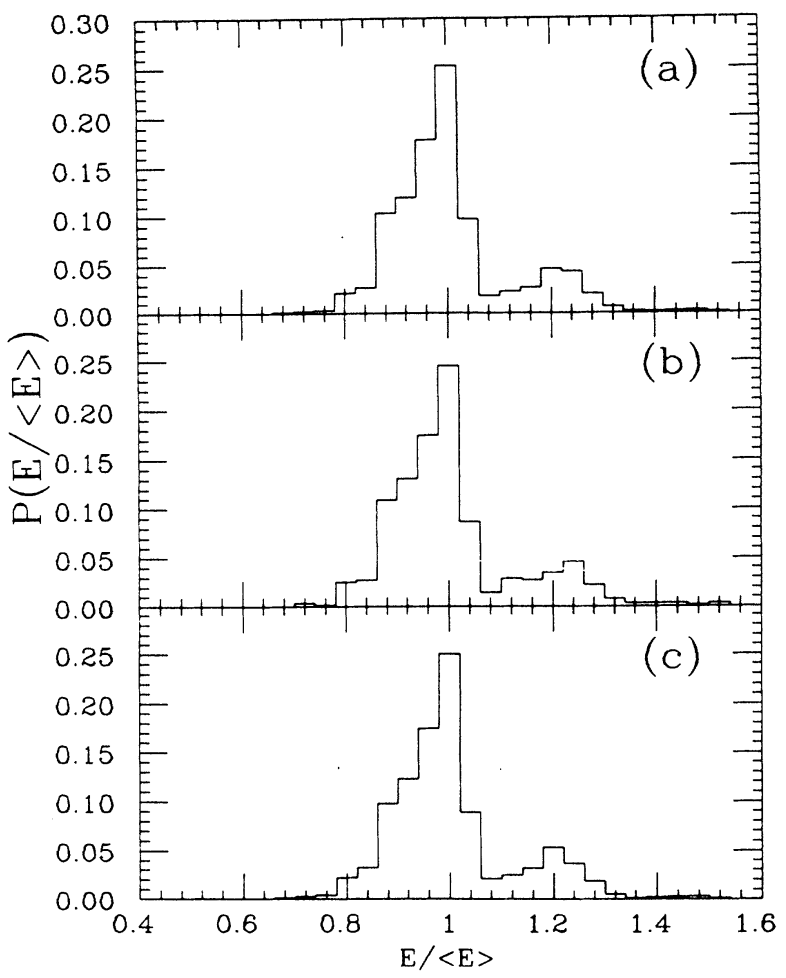

FIG. 5. Local-field distributions of uniform random media at number density 0.1 for (a) all particles inside the cavity ( $r<a-\delta$, with $a \sim 6.5$ and $\delta=0.5$ in units of lattice constant), (b) particles far away from the cavity boundary $(r<a-3.0)$, and (c) particles near the cavity boundary $(a-1.5 \leq r<a-\delta)$. The results are the average over 40 configurations. 


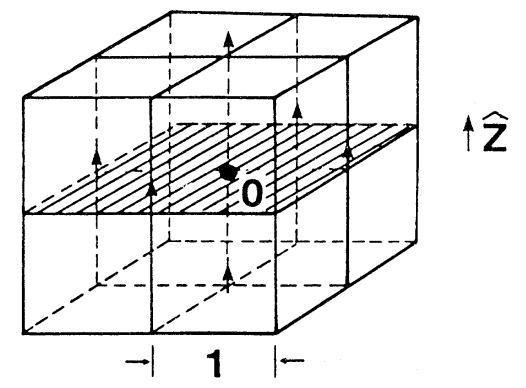

FIG. 6. Illustration of a occupied site with its nearest neighbors on a simple cubic lattice.

two groups. Group I consists of two sites on the axis parallel to $\hat{\mathbf{z}}$ through the site $O$, and group II consists of four other sites in the plane perpendicular to $\widehat{z}$. A dipole polarized along $+\hat{\mathbf{z}}$ in a group-II site produces an electric field $-\frac{1}{2} \hat{\mathbf{z}}$ at $O$ (the polarizability, lattice constant, and the applied field strength are assumed to be 1 for convenience), which differs from the value $1 \hat{\mathbf{z}}$ for a dipole in a group-I site. The double-peak character in the distribution of local-field values is therefore seen to be associated with the existence of two types of local environment relative to the applied field direction. For instance, a local configuration with only the sites in the group-I sites occupied will result in an electric field at $O$ larger than the average, whereas in the case where nearest-neighbor configurations consist of occupied sites only in the perpendicular plane the local-field value will be less than the average. In general, nearest-neighbor configurations consist of occupied sites from both groups. The probability of occurrence for the configurations and their associated local-field values at $O$ can be enumerated exactly. Suppose the occupation probability of each site is $p$, then the probability to have $n$ sites $(n \leq 2)$ in group I and $m$ sites $(m \leq 4)$ in group II occupied is $p^{n+m}(1-p)^{6-n-m}$. The total number of the configurations with $n$ dipoles in group I and $m$ dipoles in group II is $C_{2}^{n} C_{4}^{m}$, where $C_{l}^{k}=l ! /(l-k) !$. Since each configuration $(n, m)$ produces a local field $E=(n-m / 2)$ at $O$, the probability to have this field value at $O$ is given by

$$
P(E)=C_{2}^{n} C_{4}^{m} p^{n+m}(1-p)^{6-(n+m)} .
$$

Here $E$ denotes the deviation from the mean local-field value.

In Fig. 7. we show a plot of $P(E)$ given by Eq. (16) for three occupation probabilities corresponding to those shown in Fig. 3. There is a clear qualitative correspondence with the calculated double-peak distributions. In the $p=0.5$ case the single peak is indeed seen to be the merging of two peaks.

While the nearest-neighbor approximation is not sufficient to describe the percolative structures, a crude estimation can be made by substracting from Eq. (16) the term corresponding to the configuration where all nearest-neighbor sites are empty (since in percolative structures all particles are connected to their neighbors). Figure 6(d) does indeed show the pronounced double-

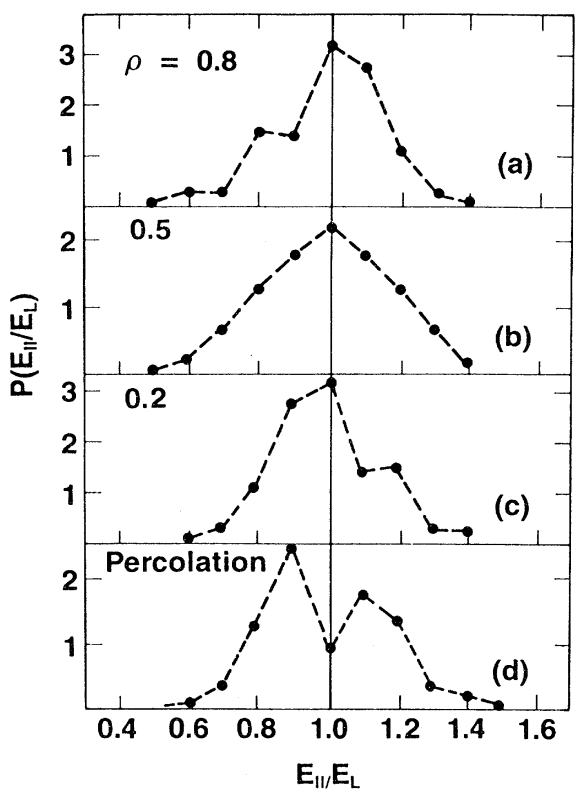

FIG. 7. Probabilistic distribution of local fields calculated with the nearest-neighbor approximation. Good qualitative agreement with Fig. 2 is clearly seen.

peak character and the accompanying increase in the distribution width as observed in Fig. 3(d). Since the value $E=n-m / 2$ depends linearly on $\alpha$, Eq. (16) also predicts that the separation between the two peaks depends linearly on the polarizability. However, our calculated results show that whereas for $\alpha<0.1$ (in units of lattice constant cubed) the peak separation is indeed linear in $\alpha$, for $\alpha>0.1$ the separation increases somewhat faster than linear. The discrepancy arises from the many-body electromagnetic interaction that tends to increase the effective polarizability. Such interactions are nonlinear in $\alpha$, as can be seen from Eqs. (9) - (15).

\section{B. The second moment of the distribution}

The discussion of Sec. III A suggests that the distribution width is sensitive to the microstructure of the system as well as to the particle polarizability. To quantify such relationships, we first define a parameter that measures the degree of local anisotropy of a random medium. For a random medium, we notice that the deviation from the Lorentz local-field value can be attributed to the dipole field inside the Lorentz cavity. A first-order estimate for the magnitude of the deviation may therefore be obtained from the parameter

$$
\eta=\left\langle\left|\sum_{j \neq i} \mathbf{D}\left(\mathbf{r}_{i}, \mathbf{r}_{j}\right)\right|^{2}\right\rangle^{1 / 2}
$$

where the dipole field $\mathbf{D}$ at $i$ is calculated by assuming ${ }^{20}$ that the dipole at every occupied site is given by a unit vector $\widehat{\mathbf{z}}$ and the summation is over all occupied sites $\mathbf{r}_{j}$ in a sufficiently large spherical cavity (so that the values of $\eta$ converge) centered at an arbitrary site $r_{i}$. From symmetry, we know that $\eta$ is zero for a cubic or spherical en- 
vironment as assumed in the Lorentz formula, and $\eta \neq 0$, in general, for random systems. Therefore $\eta$ may be regarded as a geometric parameter that measures the local anisotropy in the dipolar sense. In Fig. 8, the $\eta$ parameter is plotted as a function of number density $\rho$ for both the uniform and percolative random systems. At large densities, difference between the two random systems is small, and $\eta$ exhibits the same behavior for both. As $\rho$ decreases, the requirement for the particles to be connected makes the local structure of the percolative system much more anisotropic than the uniform case. This increased local anisotropy is reflected in the increased value of $\eta$.

In Fig. 9, the normalized standard deviation of the distribution, $\sigma=\left\langle\left(E_{\|} /\left\langle E_{L}\right\rangle\right)^{2}-1\right\rangle^{1 / 2}$, is plotted versus the $\eta$ parameter for different samples. Three different polarizabilities, $\alpha=0.05,0.1$, and 0.15 , are used in the calculation so that the $\alpha$ dependence can be explored. In each case, a least-squares fit by the equation $\sigma=a+b(\alpha) \eta$ yields an excellent linear relation between the $\sigma$ and the $\eta$ :

$$
\sigma=b(\alpha) \eta
$$

with $a \sim 10^{-2}-10^{-3}$, i.e., the intercept is close to 0 . The coefficients $b(\alpha)$ are found to be $0.909 \alpha, 0.94 \alpha$, and $1.12 \alpha$ for $\alpha=0.05,0.1$, and 0.15 , respectively. Unlike the predicted linear dependence by the nearest-neighbor analysis, $b(\alpha)$ is seen to have a slightly nonlinear dependence on $\alpha$. Equation (18) therefore states that the distribution width as measured by the standard deviation of the local field is directly proportional to the product of the $\eta$ parameter, which measures the degree of local anisotropy of a random structure, and a factor $b(\alpha)$ which is nearly linear in $\alpha$.

\section{The first moment of the distribution}

In this section, we examine the mean of the local-field distribution and its relation to the macroscopic dielectric properties of random media. The relation between the

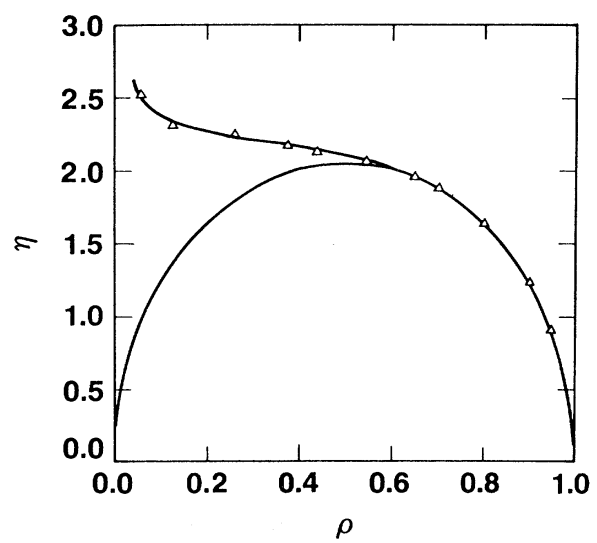

FIG. 8. Density dependence of the local anisotropy parameter $\eta$ for both the uniform and the percolative random media. The open triangles denote the simulation results for the percolative random media. The difference in the degree of local anisotropy between these two types of system is demonstrated by the $\eta$ parameter in the low-density region.

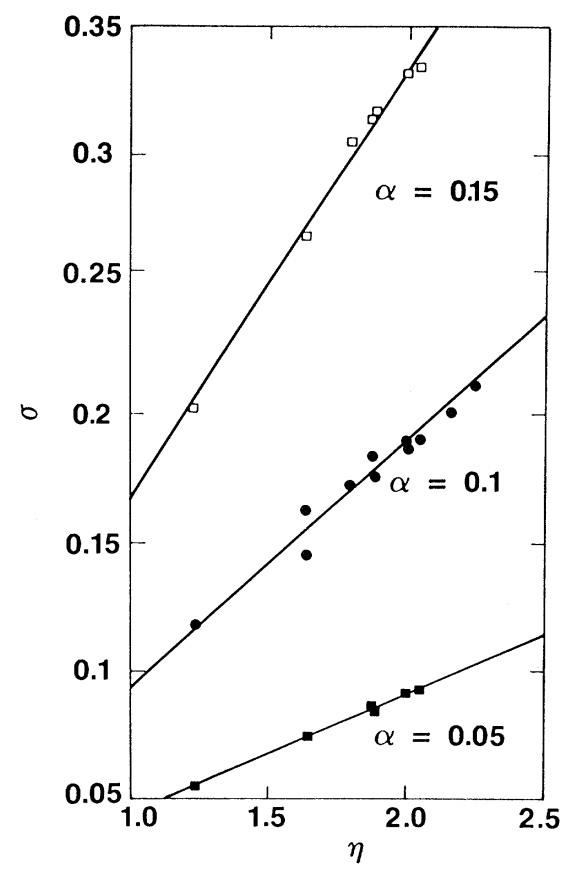

FIG. 9. Standard deviation of the local-field distribution for three different polarizabilities plotted as a function of $\eta$ defined in the text. Solid triangles denote the results for percolative structures where the value of $\sigma$ for each point is evaluated from distributions calculated with 3000-6000 particles; all other data points are for uniform random systems with each $\sigma$ value evaluated from distributions containing 1500-3000 particles. The solid lines are the least-squares fits to the equation $\sigma=a+b \eta$.

dielectric constant $\epsilon$ and the mean of the local field $\left\langle E_{L}\right\rangle$ is expressed by Eq. (10). For comparison, let us express

$$
\begin{aligned}
\left\langle E_{L}\right\rangle & =E_{\text {Lorentz }}+\left(\left\langle E_{L}\right\rangle-E_{\text {Lorentz }}\right) \\
& =E_{\text {Lorentz }}+\Delta E .
\end{aligned}
$$

Equation (10) can be rewritten as

$$
\begin{aligned}
\frac{\epsilon-1}{4 \pi} & =\rho \alpha\left(E_{\text {Lorentz }}+\Delta E\right) \\
& =\rho \alpha E_{\text {Lorentz }}\left(1+\frac{\Delta E}{E_{\text {Lorentz }}}\right) .
\end{aligned}
$$

By defining $E_{\text {Lorentz }}=(\epsilon+2) / 3$ in units of the average macroscopic field and substituting it into Eq. (19), an equation for the dielectric constant $\epsilon$ is obtained:

$$
\frac{\epsilon-1}{\epsilon+2}=\frac{4 \pi}{3} \rho \alpha(1+S),
$$

where $S=\Delta E / E_{\text {Lorentz }}$ is the correction factor to the CM relation. For a fully occupied simple cubic lattice, $S=0$, and the CM relation is rigorously valid.

Before we show the results of $S$ for random systems, we note that Eq. (20) is in the form of Kirkwood-Yvon (KY) theory derived by using formal statistical mechanics. In the KY theory the leading term for $S$ may be expressed in terms of the integrals over the many-body correlation functions: 


$$
S=\alpha^{2}\left(8 \pi \rho \int g_{2}(r) r^{-4} d r+2 \rho^{2} \int\left[g_{3}(r, s)-g_{2}(r) g_{2}(s)\right] r^{-3} s^{-3} P_{2}(\cos \theta) d^{3} r d^{3} s\right]+O\left(\alpha^{3}\right),
$$

where $g_{2}$ and $g_{3}$ are pair and three-body correlation functions, $P_{2}(\cos \theta)$ is the second Legendre polynomial, and $\theta$ is the angle between $\mathbf{r}$ and $\mathbf{s}$. Even for this leading-order correction, however, one already encounters the threebody correlation function. The simplifying superposition approximation $^{21}$ used to express $g_{3}$ as products of $g_{2}$ 's will be seen to severely limit the accuracy in the evaluation of $S$. We wish to show that, to order $\alpha^{2}$, the correction $S^{(2)}$ is directly related to the $\eta$ parameter as defined in Sec. III B. To see this connection, ${ }^{22}$ we take the origin of $\mathbf{r}$ and $\mathbf{s}$ in Eq. (21) to be at the center of a spherical sample. The integral of the $g_{2}(r) g_{2}(s)$ term then vanishes and the integral of the $g_{3}(r, s)$ term can be written in terms of the dipolar tensor as

$$
\alpha^{2}\left\langle\widehat{\mathbf{z}} \cdot \sum_{j \neq k} \overleftrightarrow{\mathbf{T}}_{i j} \cdot \overleftrightarrow{\mathbf{T}}_{i k} \cdot \widehat{\mathbf{z}}\right\rangle
$$

where $\overleftrightarrow{\mathbf{T}}_{i j}$ is defined in Eq. (8) and $\widehat{\mathbf{z}}$ is the unit vector of the applied electric field. The first integral in Eq. (21) can also be written in terms of the dipolar tensor as

$$
\alpha^{2}\left\langle\widehat{\mathbf{z}} \cdot \sum_{j} \overleftrightarrow{\mathbf{T}}_{i j} \cdot \overleftrightarrow{\mathbf{T}}_{i j} \cdot \widehat{\mathbf{z}}\right\rangle
$$

By adding (22) to (23), we get

$$
\begin{aligned}
S^{(2)} & =\alpha^{2}\left\langle\widehat{\mathbf{z}} \cdot\left(\sum_{j} \overleftrightarrow{\mathbf{T}}_{i j}\right) \cdot\left(\sum_{k} \overleftrightarrow{\mathbf{T}}_{i k}\right) \cdot \widehat{\mathbf{z}}\right\rangle \\
& =\alpha^{2} \eta^{2} \geq 0 .
\end{aligned}
$$

Therefore $S^{(2)}$ can be obtained by the result of a direct Monte Carlo calculation of $\eta$ presented in Sec. III B, thus avoiding the calculation of correlation functions. (Alder, Weis, and Strauss ${ }^{23}$ had performed such a calculation at only one number density $\rho=0.884$.)

Since our calculations are carried out on a lattice, comparison of our results with those in the literature requires the removal of the lattice effect. To this end, we define a constant $\beta^{2}=(2 \pi / 3) B \alpha^{2}$, where

$$
B=\rho \int \widehat{\mathbf{z}} \cdot \overleftrightarrow{\mathbf{T}}_{i j} \cdot \overleftrightarrow{\mathbf{T}}_{j i} \cdot \hat{\mathbf{z}} e^{-\phi_{i j} / k T} d \mathbf{r}_{i j}
$$

In Eq. (25), $\widehat{\mathbf{z}}$ is an unit directional vector, $\phi_{i j}$ is the interaction potential between particles $i$ and $j, k$ is the Boltzmann constant, and $T$ denotes temperature. For a system of hard spheres, $\phi_{i j}=0$ for $r_{i j}>d$ and $\phi_{i j}=\infty$ for $r_{i j} \leq d$, where $d$ is the diameter of a sphere. Substituting this $\phi_{i j}$ into Eq. (25) and performing the integration yields $(8 \pi / 3) d^{-6}$ for the system of hard spheres. Here $\rho d^{3}=1$. The same integration is also performed for point particles on the simple cubic lattice. In this case, the integration is replaced by summation,

$$
B=\left\langle\sum_{j} \hat{\mathbf{z}} \cdot \overleftrightarrow{\mathbf{T}}_{i j} \cdot \overleftrightarrow{\mathbf{T}}_{j i} \cdot \hat{\mathbf{z}}\right\rangle,
$$

which yields $B=16.8 d^{-6}$ for $\rho d^{3}=1$ [where $d(=1)$ is our lattice constant]. This value is about twice the value calculated for the hard sphere system. Division of $S$ by $\beta^{2}$ removes the leading-order dependence on $\alpha$ and the underlying lattice and makes possible direct comparison with the KY theory.

In Fig. 10 the Monte Carlo results of $S^{(2)} / \beta^{2}$ for both the uniform and the percolative random systems are plotted as a function of $\rho$ in solid lines. Values of $S / \beta^{2}$ calculated from the local-field distribution with three different polarizabilities are plotted in symbols. Kirkwood's analytic result for a system of low-density hard spheres is also plotted in a dashed line for comparison. The good agreement of $S^{(2)}$ with the calculated values of $S$ demonstrates that $S^{(2)}$ is the major fraction of $S$. Several points should be noted from Fig. 10. First, in all our calculations $S \geq 0$. This is consistent with the $\mathrm{KY}$ results since the $S^{(2)}$ given by Eq. (24) is non-negative. In a different context, Hellwarth ${ }^{24}$ has also shown that the $\epsilon$ calculated from the CM relation is in fact a lower bound for the dielectric constant, which implies $S \geq 0$. A curious implication of the inequality $\left\langle E_{L}\right\rangle \geq E_{\text {Lorentz }}$ is that if an initially simple cubic material is randomized, then the net absorption of the material would increase because the mean of the local fields does not average to the same value as before, but would always be larger. Furthermore, for two materials with the same density the materi-

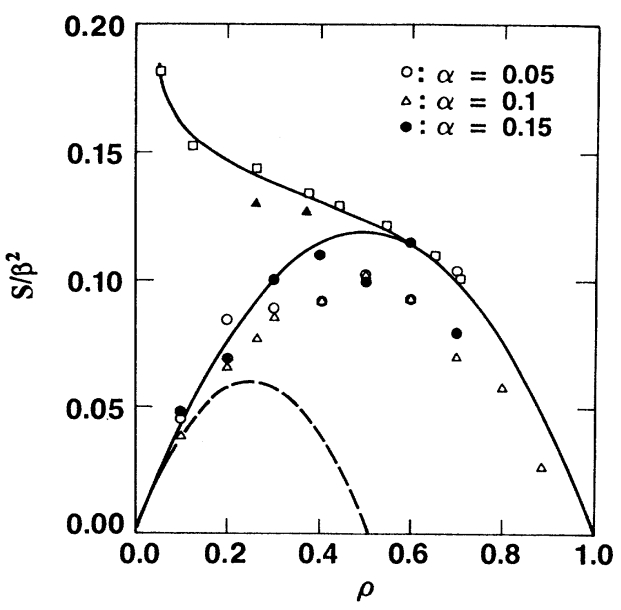

FIG. 10. Correction to the Clausius-Mossotti relation plotted as a function of the particle number density. Symbols (except the open squares) represent the results obtained using the generalized Onsager approach with the same statistics as that for Fig. 9. Solid triangles are for percolative random structures. Solid lines denote the fit to Monte Carlo results of $S^{(2)}=\alpha^{2} \eta^{2}$ from the KY theory evaluated as the average of 30000-100000 particles. In the simulation of percolative random structures (open squares), the correlation lengths, in descending order, are (in units of lattice constant) 176, 23, 6.4, 3.4, 2.3, 1.5, and 1.1. Three different polarizabilities are used for the calculation of homogeneous random systems. The dashed line represents the analytic result of Kirkwood. 
al with more stringlike structures (such as a fractal aggregate) would yield bigger absorption since the $S^{(2)} \propto \eta^{2}$, and $\eta$ is a measure of local anisotropy. It follows that the $S$ of a percolative random system is always larger than or equal to that of the uniform random system at any fixed density. Second, for Kirkwood's analytic solution of $S^{(2)}$, the agreement with our numerical calculation is indeed excellent at low densities for uniform random systems. At high densities, however, the Kirkwood solution differs significantly from both the calculated $S$ and the Monte Carlo evaluation of $S^{(2)}$ due to the inaccuracy of the analytic solution utilizing the superposition approximation. In percolative random structures the Kirkwood solution is seen to be grossly inadequate even at low densities.

The effect of extended spatial correlation in a random system is clearly visible through the distinct behaviors of the correction factor as a function of density. Whereas the uniform random media has the maximum CM correction at $\rho=0.5$, percolative structures with extended correlations show a monotonic increase in the value of $S$ as the correlation length increases. A finite limiting value of $S$ is predicted for $\rho=0$ (and $\xi \rightarrow \infty$ ), which would be that of a percolation cluster. A upper bound of this limiting value is calculated from a straight infinite chain of dipoles, which yields $S_{\max }^{(2)} / \beta^{2} \leq 0.35$. Since this increase in $S$ at low densities arises from increased local anisotropy, we expect the local-field distribution for aggregates or growth structures to have large variances. The Lorentz local-field value would therefore be a poor approximation in these systems.

In conclusion, since $S \simeq S^{(2)}=\alpha^{2} \eta^{2}$ and $\sigma \cong \alpha \eta$, we deduce the approximate general relation

$$
S \propto \sigma^{2} .
$$

That is, if the width of the distribution is zero, then $\left\langle E_{L}\right\rangle$ must be equal to $E_{\text {Lorentz }}$. As $\left\langle E_{L}\right\rangle$ increases away from $E_{\text {Lorentz }}$ the distribution width increases correspondingly. This prediction should be experimentally verifiable by comparing, for example, the broadening of spectral lines in a gas due to the Stark effect with the deviation of the dielectric constant from the CM value. ${ }^{25}$ We have also systematically checked the $\alpha$ dependence of $S$ and found it not strictly quadratic at large $\alpha$ values. This is consistent with the earlier observation about the distribution width dependence of $\sigma$.

\section{EFFECTS OF UNDERLYING LATTICE AND FINITE PARTICLE SIZE}

Since our random systems are generated on a simple cubic lattice, one may first ask whether our results would be affected by the orientation of the applied $\mathbf{E}$ field relative to the lattice. For the simple cubic lattice, it is easy to see that the orientation should have no effect because we can always decompose the applied field into components that are parallel to the lattice axes. Since the problem is linear for the electric field, we can apply the superposition principle. The equivalence of the three lattice axis directions immediately implies that the resultant statistical properties are identical to what we have obtained. But suppose now we consider a different lattice, how much of the distribution characteristics would be affected by the underlying lattice? To answer this question, we first note that the applied field direction constitutes a special direction for each local configuration in a random structure as noted before. In relation to this special direction, one type of local environment has more particles near the axis along the applied field direction than the plane perpendicular to the field direction. These local configurations produce local fields above the mean. The other type of local environment has more sites occupied near the plane perpendicular to the field direction and thereby yields values less than the mean. Unless the local environment is always perfectly spherical or cubic, the distribution of the local-field values is expected to fall into one of the two local environment types described above. The double-peak character should therefore be generic to random dielectrics. However, since the separation (or width $\sigma$ ) between the two peaks depends on $\alpha$, the two peaks may not always be visible at low $\alpha$ values. In Fig. 11, we plotted the local-field distributions calculated for random systems with $\rho=0.2$ generated on a bcc lattice. Two different polarizabilities were used. While the distribution for $\alpha=0.1$ shows almost a single peak, the two peaks become visibly separated when $\alpha$ is increased to 0.15 . With the results shown above, we conclude that the double-peak distribution is generic to local-field distributions. However, for an off-lattice random medium the visibility of these two peaks depends on the density, the number of nearest neighbors, and the polarizability.

Since all molecules and atoms have hard cores, it is known that at moderate to high densities the short-range order of hard spheres can be characterized by partial lo-

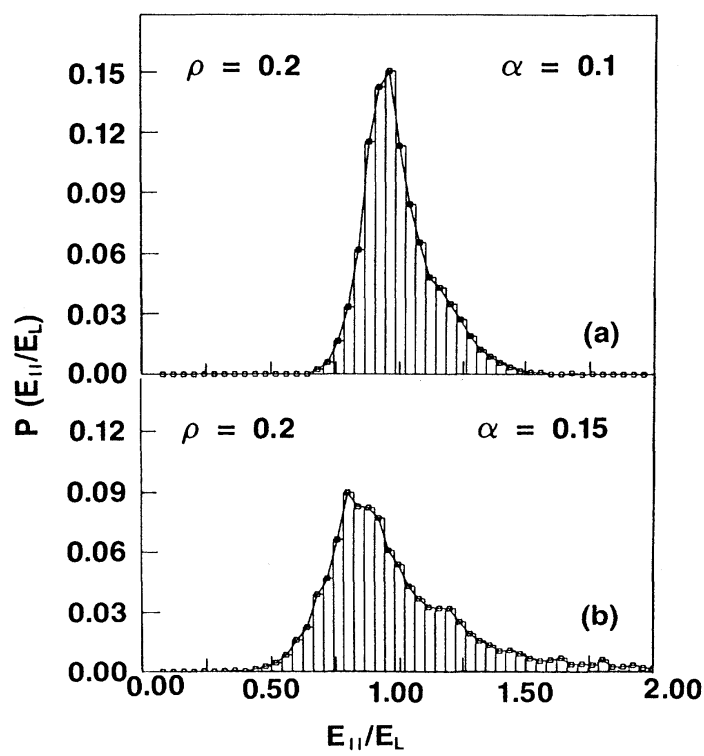

FIG. 11. Local-field distributions for a uniform random system, generated on a bcc lattice, plotted as a function of $E_{\|} / E_{L}$. (a) is obtained with the particle polarizability $\alpha=0.1$. In (b), $\alpha=0.15$. As $\alpha$ increases, the two-peak character of the distribution becomes apparent. 
cal (latticelike) order. Thus even for liquids the discussion above concerning the lattice effects is relevant.

The local-field distributions shown in the preceding sections are all calculated with point dipoles. While the assumption of a point dipole is often a good approximation at the atomic level and for macroscopic particles which are widely separated, effects of finite particle size could become important as the particle separation decreases so that high-order multipole interactions become comparable to dipole interaction. Since a straightforward inclusion of the multipole-interaction effect may pose formidable calculational difficulties, we suggest that the problem may be made tractable by dividing it into two steps. Since multipole interaction is short ranged as compared to the dipole interaction, one may regard the short-range, high-order multipole interaction as having the net effect of renormalizing the vacuum polarizability of a particle to a new value that is more appropriate for its local environment. The resultant $\alpha$ could be a tensoral quantity. An approximate solution to the local-field problem for finite-sized particles may now be obtained by treating the particles again as pointlike particles but with the renormalized polarizability. This program has indeed been carried out for the calculation of optical properties of fractal colloidal clusters with gratifying results. ${ }^{25}$

\section{CORRECTION TO EFFECTIVE MEDIUM THEORIES}

In Sec. III, we have seen that fluctuation of local fields in random systems, as characterized by their distribution function, are very sensitive to the microstructure of the system. For a percolative random medium the Lorentz local field and its prediction on the dielectric constant are by no means accurate. Since a random system in general possesses a correlated structure at scales less than $\xi$, the effective-medium theories widely used to predict the dielectric behaviors may have to be modified so as to incorporate such microstructural effects.

For illustration, let us take the widely used MaxwellGarnett ${ }^{3}$ theory as an example. The Maxwell-Garnett formula can be derived in many equivalent ways with the assumption of a homogeneous local environment. The traditional derivation is to substitute the electrostatic polarizability $\alpha=r_{0}^{3}\left(\epsilon_{m}-1\right) /\left(\epsilon_{m}+2\right)$ for a spherical particle, where $\epsilon_{m}$ is the dielectric constant of an inclusion particle and $r_{0}$ its radius, into the $\mathrm{CM}$ relation. This yields the Maxwell-Garnett formula

$$
\frac{\epsilon-1}{\epsilon+2}=f \frac{\epsilon_{m}-1}{\epsilon_{m}+2},
$$

where $f=(4 \pi / 3) \rho r_{0}^{3}$ is the volume fraction of the particle inclusion. Equation (28) has been used as the basic equation for the description of optical properties of composites. The assumption of a homogeneous local environment in the CM relation obviously neglects the microstructure and the local-field fluctuations in a random system.

These effects can now be included by using our equation (20). By using the same electrostatic polarizability of the particle inclusion, Eq. (20) can be written as

$$
\frac{\epsilon-1}{\epsilon+2}=f[1+S(f, \alpha, \xi)] \frac{\epsilon_{m}-1}{\epsilon_{m}+2} .
$$

We thus get an extension of the Maxwell-Garnett formula which can serve as the effective-medium theory for aggregated or fractal structures. For thin granular metal films, the experimental observation of the red shift and broadening of the absorption peaks can be well accounted for by Eq. (29). Our calculations on these aspects and the comparison to other mean-field theories will be reported elsewhere.

\section{CONCLUDING REMARKS}

We have presented a direct extension of the Lorentz theory of local field to random dielectrics. Quantitative relationships between the local-field distribution characteristics and the geometric and dielectric properties of a random system are obtained. It would be very interesting to see an analytic theory for the distribution functions. Such a theory would necessarily have to incorporate the effects of the microstructure as seen from our results. Also, while our results are obtained for nonpolar systems, the formulation may be further developed to treat polar systems. In this case, however, numerical calculation needs to have an efficient Monte Carlo algorithm to handle the thermodynamics. A set of new phenomena, such as a spin-glass-like state, is anticipated. Investigations in this direction are currently underway.

\section{APPENDIX}

In this appendix, we derive a general expression for the reaction field $\mathbf{R}\left(\mathbf{r}_{i}, \mathbf{r}_{j}\right)$ with arbitrary $\mathbf{r}_{i}$ and $\mathbf{r}_{j}$. To do this, we first consider the electric field at $\mathbf{r}$ produced by a dipole $\mathbf{p}$ at $\mathbf{r}_{j}$ in vacuum. The electric potential $\phi_{0}$ satisfies the Poisson equation:

$$
\nabla^{2} \phi_{0}(\mathbf{r})=-4 \pi \rho(\mathbf{r}),
$$

where $\rho(\mathbf{r})$ is the free charge density. In terms of Green's function, the general solution of Eq. (A1) is

$$
\phi_{0}(\mathbf{r})=\int_{V \rightarrow \infty} \rho\left(\mathbf{r}^{\prime}\right) G\left(\mathbf{r}, \mathbf{r}^{\prime}\right) d^{3} \mathbf{r}^{\prime},
$$

where the Green's function $G\left(\mathbf{r}, \mathbf{r}^{\prime}\right)$ is given by

$$
\begin{aligned}
G\left(\mathbf{r}, \mathbf{r}^{\prime}\right) & =\frac{1}{\left|\mathbf{r}-\mathbf{r}^{\prime}\right|} \\
& =4 \pi \sum_{l=0}^{\infty} \sum_{m=-l}^{l} \frac{1}{2 l+1} \frac{r_{<}^{l}}{r_{>}^{l+1}} Y_{l m}^{*}\left(\theta^{\prime}, \phi^{\prime}\right) Y_{l m}(\theta, \phi) .
\end{aligned}
$$

Here $r_{<}=\min \left(r^{\prime}, r\right), r_{>}=\max \left(r^{\prime}, r\right)$, and $Y_{l m}(\theta, \phi)$ is the normalized spherical harmonic function. For a polarizable medium with polarization $\mathbf{P}, \rho(\mathbf{r})=-\boldsymbol{\nabla} \cdot \mathbf{P}$. Therefore

$$
\rho(\mathbf{r})=-\nabla \cdot\left[\mathbf{p} \delta\left(\mathbf{r}-\mathbf{r}_{j}\right)\right]=-\mathbf{p} \cdot \boldsymbol{\nabla} \delta\left(\mathbf{r}-\mathbf{r}_{j}\right) .
$$

Substitution of Eq. (A4) into Eq. (A2) yields the electric 
potential in region $r>r_{j}$ :

$$
\phi_{0}(\mathbf{r})=\sum_{l=0}^{\infty} \sum_{m=-l}^{l} C_{l m}\left(\mathbf{r}_{j}\right) \frac{1}{r^{l+1}} Y_{l m}(\theta, \phi),
$$

with

$$
C_{l m}\left(\mathbf{r}_{j}\right)=-\mathbf{p} \cdot\left[\frac{4 \pi}{2 l+1} \int \nabla \delta\left(\mathbf{r}-\mathbf{r}_{j}\right) r^{l} Y_{l m}^{*}(\theta, \phi) d^{3} r\right] .
$$

By noticing the identity

$$
\left[\boldsymbol{\nabla} \delta\left(\mathbf{r}-\mathbf{r}_{j}\right)\right] g(\mathbf{r})=\nabla\left[\delta\left(\mathbf{r}-\mathbf{r}_{j}\right) g(\mathbf{r})\right]-\delta\left(\mathbf{r}-\mathbf{r}_{j}\right)[\boldsymbol{\nabla} g(\mathbf{r})],
$$

the integral of Eq. (A6) can be simplified if we let $g(\mathbf{r})$ denote $r^{l} Y_{l m}^{*}(\theta, \phi)$. Then the integration of the first term $\boldsymbol{\nabla}\left[\delta\left(\mathbf{r}-\mathbf{r}_{j}\right) g(\mathbf{r})\right]$ vanishes for $\mathbf{r}_{j} \neq 0$. Integration of the second term of Eq. (A7) simply replaces the argument of $g(\mathbf{r})$ by $\mathbf{r}_{j}$, a consequence of the $\delta$-function property. Therefore

$$
C_{l m}\left(\mathbf{r}_{j}\right)=-\frac{4 \pi}{2 l+1} \mathbf{p} \cdot \nabla\left[r_{j}^{l} Y_{l m}^{*}\left(\theta_{j}, \phi_{j}\right)\right] .
$$

Now let us place the dipole $\mathbf{p}$ at $\mathbf{r}_{j}$ inside a cavity of radius $a$. The electric potentials inside, $\phi_{\text {in }}$, and outside, $\phi_{\text {out }}$, the cavity satisfy the Laplace equation:

$$
\begin{aligned}
& \nabla^{2} \phi_{\text {in }}(\mathbf{r})=0, \text { for } r_{j}<r<a, \\
& \nabla^{2} \phi_{\text {out }}(\mathbf{r})=0, \text { for } r>a .
\end{aligned}
$$

Their general solution is in the form of

$$
\begin{aligned}
& \phi_{\text {in }}(\mathbf{r})=\sum_{l=0}^{\infty} \sum_{m=-l}^{l}\left(A_{l m} r^{l}+B_{l m} r^{-l-1}\right) Y_{l m}(\theta, \phi), \\
& \phi_{\text {out }}(\mathbf{r})=\sum_{l=0}^{\infty} \sum_{m=-l}^{l}\left(C_{l m} r^{l}+D_{l m} r^{-l-1}\right) Y_{l m}(\theta, \phi),
\end{aligned}
$$

where $A_{l m}, B_{l m}, C_{l m}$, and $D_{l m}$ are coefficients to be determined by the following boundary conditions:

$$
\begin{aligned}
& \phi_{\text {in }}(\mathbf{r})=\phi_{\text {out }}(\mathbf{r}), \text { at } r=a, \\
& \frac{\partial \phi_{\text {in }}(\mathbf{r})}{\partial r}=\epsilon \frac{\partial \phi_{\text {out }}(\mathbf{r})}{\partial r}, \text { at } r=a, \\
& \phi_{\text {out }}(\mathbf{r}) \rightarrow 0, \text { for } r \rightarrow \infty, \\
& \phi_{\text {in }}(\mathbf{r})=\phi_{\text {out }}(\mathbf{r})=\phi_{0}(\mathbf{r}), \text { when } \epsilon=1 .
\end{aligned}
$$

Boundary condition (A13d) simply states that when the cavity is absent $(\epsilon=1)$ the electric potentials should be identical to the solution of the dipole in the vacuum, Eq. (A5). The boundary conditions (A13b)-(A13d) completely determine the expansion coefficients, and the solutions are

$$
\phi_{\text {in }}\left(\mathbf{r}, \mathbf{r}_{j}\right)=\sum_{l=0}^{\infty} \sum_{m=-l}^{l}\left[\frac{(\epsilon-1)(l+1)}{l+(l+1) \epsilon} a^{-2 l-1} r^{l}+r^{-l-1}\right] C_{l m}\left(\mathbf{r}_{j}\right) Y_{l m}(\theta, \phi),
$$

and

$$
\phi_{\text {out }}\left(\mathbf{r}, \mathbf{r}_{j}\right)=\sum_{l=0}^{\infty} \sum_{m=-l}^{l} \frac{(2 l+1)}{l+(l+1) \epsilon} r^{-l-1} C_{l m}\left(\mathbf{r}_{j}\right) Y_{l m}(\theta, \phi),
$$

where $C_{l m}\left(\mathbf{r}_{j}\right)$ is given by Eq. (A8). The part of the potential in $\phi_{\text {in }}$ that arises from the polarization of the outside medium by the dipole is simply the difference of $\phi_{\text {in }}$ and the dipolar field $\phi_{0}$ in vacuum, i.e.,

$$
\phi_{R}\left(\mathbf{r}, \mathbf{r}_{j}\right)=\phi_{\text {in }}\left(\mathbf{r}, \mathbf{r}_{j}\right)-\phi_{0}\left(\mathbf{r}, \mathbf{r}_{j}\right)=-\sum_{l=0}^{\infty} \sum_{m=-l}^{l} \frac{(\epsilon-1)(l+1)}{l+(l+1) \epsilon} a^{-2 l-1} r^{l} C_{l m}\left(\mathbf{r}_{j}\right) Y_{l m}(\theta, \phi) .
$$

So the reaction field at $\mathbf{r}$ is

$$
\begin{aligned}
\mathbf{R}\left(\mathbf{r}, \mathbf{r}_{j}\right)=-\nabla \phi_{R}\left(\mathbf{r}, \mathbf{r}_{j}\right) & =\sum_{l=0}^{\infty} \sum_{m=-l}^{l} \frac{(\epsilon-1)(l+1)}{l+(l+1) \epsilon} a^{-2 l-1} C_{l m}\left(\mathbf{r}_{j}\right) \nabla\left[r^{l} Y_{l m}(\theta, \phi)\right] \\
& =\alpha \sum_{l=0}^{\infty} \sum_{m=-l}^{l} c_{l m}(\epsilon)\left[\mathbf{q}^{*}\left(l, m, \mathbf{r}_{j}\right) \cdot \mathbf{p}\right] \mathbf{q}(l, m, \mathbf{r})
\end{aligned}
$$

where $\mathbf{p}=\alpha \mathbf{E}_{L}$, and

$$
\begin{aligned}
& \mathbf{q}\left(l, m, \mathbf{r}_{i}\right)=\nabla\left[\left(\frac{\left|\mathbf{r}_{i}\right|}{a}\right)^{l} Y_{l m}\left(\theta_{i}, \phi_{i}\right)\right], \\
& c_{l m}(\epsilon)=\frac{4 \pi}{2 l+1} \frac{(\epsilon-1)(l+1)}{l+(l+1) \epsilon} \frac{1}{a} .
\end{aligned}
$$

Since there is no free charge in region $0 \leq r \leq r_{j}$, the above solution of the reaction field is also valid in this region.

The total dipole moment of the cavity can be obtained from the total electric potential $\phi_{\text {out }}^{T}$ in the outside medium when an external field is applied to the medium. $\phi_{\text {out }}^{T}$ consists of three contributions: (1) the $\phi_{\text {out }}$ of Eq. (A15) 
obtained when the applied field is absent, (2) the applied field itself, and (3) the induced field of the cavity due to the polarization of the cavity wall by the applied field. Therefore,

$$
\phi_{\text {out }}^{T}(\mathbf{r})=\sum_{j=1}^{N} \phi_{\text {out }}\left(\mathbf{r}, \mathbf{r}_{j}\right)-E r \cos \theta-\frac{\epsilon-1}{2 \epsilon+1} E a^{3} \frac{\cos \theta}{r^{2}} .
$$

The total dipole moment $d$ of the cavity is thus obtained by picking out the $l=1, m=0$ component of the $\phi_{\text {out }}\left(\mathbf{r}, \mathbf{r}_{j}\right)$ :

$$
d=-\frac{\epsilon-1}{2 \epsilon+1} E a^{3}+\frac{3}{2 \epsilon+1} \sum_{j=1}^{N} C_{10}\left(\mathbf{r}_{j}\right)
$$

${ }^{*}$ Present address: Brown University, Box H, Providence, RI 02912.

${ }^{1}$ See, for example, C. Kittle, Introduction for Solid State Physics, 5th ed. (Wiley, New York, 1952).

${ }^{2}$ H. A. Lorentz, The Theory of Electrons (B. G. Teubner, Leipzig, 1909; reprint: Dover, New York, 1952).

${ }^{3}$ See R Landauer, in Electrical Transport and Optical Properties of Inhomogeneous Media (Ohio State University, 1977), Proceedings of the First Conference on the Electrical Transport and Optical Properties of Inhomogeneous Media, AIP Conf. Proc. No. 40, edited by J. C. Garland and D. B. Tanner (AIP, New York, 1978), p. 2.

4J. G. Kirkwood, J. Chem. Phys. 4, 592 (1936).

$5 \mathrm{~J}$. Yvon, Recherches sur la Théorie Cinétique des Liquides II (Hermann, Paris, 1937).

${ }^{6}$ W. F. Brown, Jr., J. Chem. Phys. 23, 154 (1955).

${ }^{7}$ J. de Boer, F. van der Maesen, and C. A. van Seldam, Physica 19, 265 (1955).

${ }^{8}$ U. Geigenmüuer and P. Mazar, Physica 136A, 316 (1986).

${ }^{9}$ P. Debye, Polar Molecules (The Chemical Catalog Company, Inc., New York, 1929), p. 27.

${ }^{10}$ H. Fröhlich, Theory of Dielectrics, 2nd ed. (Oxford University Press, London, 1958).

${ }^{11}$ J. H. van Vleck, J. Chem. Phys. 5, 320 (1937); 5, 556 (1937).

${ }^{12}$ L. Onsager, J. Am. Chem. Soc. 58, 1486 (1936).

${ }^{13}$ P. Sheng and Z. Chen, Phys. Rev. Lett. 60, 227 (1988).

${ }^{14} \mathrm{R}$. Zallen, The Physics of Amorphous Solids (Wiley, New York, 1983).
${ }^{15}$ See, for example, P. Sheng, in Homogenization and Effective Moduli of Materials and Media, edited by J. L. Eriksen (Springer-Verlag, New York, 1986), Vol. 1.

16J. D. Jackson Classical Electrodynamics, 2nd ed. (Wiley, New York, 1975).

${ }^{17}$ D. Stauffer, Introduction to Percolation Theory (Taylor and Francis, London, 1985).

${ }^{18}$ B. B. Mandelbrot, The Fractal Geometry of Nature (Freeman, San Francisico, 1982).

${ }^{19}$ Since the local field is calculated on the occupied site only and the polarizable particle has no internal states such as that for the Ising spin, the double-peak character here should be distinguished from those model behaviors which originate from different types of sites or internal states.

${ }^{20}$ This assumption is inconsistent with the actual situation, where the dipole moments are different on different sites. However, the $\eta$ parameter is constructed as a geometric parameter. As such, it is successful in indicating the degree of local anisotropy.

${ }^{21}$ J. G. Kirkwood, J. Chem. Phys. 5, 300 (1935); J. G. Kirkwood and E. M. Boggs, ibid. 10, 392 (1942).

${ }^{22}$ G. Stell and G. S. Rushbrooke, Chem. Phys. Lett. 24, 531 (1974).

${ }^{23}$ B. L. Alder, J.-J. Weis, and H. L. Strauss, Phys. Rev. A 7, 281 (1973).

${ }^{24}$ R. W. Hellwarth, Phys. Rev. 152, 152 (1966).

${ }^{25}$ Z. Chen and P. Sheng, Phys. Rev. B 39, 9816 (1989). 